\title{
Retracted: Research on Comprehensive Evaluation of Economic Management Performance Based on Improved Fuzzy Clustering Algorithm
}

\author{
Security and Communication Networks
}

Received 18 November 2022; Accepted 18 November 2022; Published 7 December 2022

Copyright (C) 2022 Security and Communication Networks. This is an open access article distributed under the Creative Commons Attribution License, which permits unrestricted use, distribution, and reproduction in any medium, provided the original work is properly cited.

Security and Communication Networks has retracted the article titled "Research on Comprehensive Evaluation of Economic Management Performance Based on Improved Fuzzy Clustering Algorithm" [1] due to concerns that the peer review process has been compromised.

Following an investigation conducted by the Hindawi Research Integrity team [2], significant concerns were identified with the peer reviewers assigned to this article; the investigation has concluded that the peer review process was compromised. We therefore can no longer trust the peer review process, and the article is being retracted with the agreement of the editorial board.

\section{References}

[1] S. Xian, "Research on Comprehensive Evaluation of Economic Management Performance Based on Improved Fuzzy Clustering Algorithm," Security and Communication Networks, vol. 2022, Article ID 8578138, 9 pages, 2022.

[2] L. Ferguson, "Advancing Research Integrity Collaboratively and with Vigour," 2022, https://www.hindawi.com/post/ advancing-research-integrity-collaboratively-and-vigour/. 


\title{
Research on Comprehensive Evaluation of Economic Management Performance Based on Improved Fuzzy Clustering Algorithm
}

\section{WILEY $\mid$ OHindawi}

\begin{abstract}
Shi Xian
School of Economics and Management, University of Chinese Academy of Sciences, Beijing 201802105, China

Correspondence should be addressed to Shi Xian; 201802105@stu.ncwu.edu.cn

Received 13 January 2022; Revised 26 January 2022; Accepted 3 February 2022; Published 8 March 2022

Academic Editor: Muhammad Arif

Copyright () 2022 Shi Xian. This is an open access article distributed under the Creative Commons Attribution License, which permits unrestricted use, distribution, and reproduction in any medium, provided the original work is properly cited.

In order to solve the problems of low recall rate and precision rate, high error rate, and long evaluation time in traditional evaluation methods, a comprehensive evaluation of economic management performance based on an improved fuzzy clustering algorithm is designed. The improved magnetic optimization algorithm was used to optimize the fuzzy C-mean algorithm, the improved fuzzy clustering algorithm was completed, and the improved fuzzy clustering algorithm was used to mine the economic management performance data. Using data mining findings and AHP's weighting formula, a complete method for evaluating economic management effectiveness was developed. The BP neural network was improved using a genetic algorithm based on the index weight calculation findings, and the full-assessment model of economic management performance was constructed. Using this approach, it is possible to accurately and quickly assess the economic management performance of a company with a high rate of recall and accuracy; the error rate of a thorough assessment ranges between -3 percent and 4 percent; the average duration for an assessment is 0.81 seconds.
\end{abstract}

\section{Introduction}

With the deepening of economic and political system reform, the comprehensive evaluation of economic management performance has become an important agenda of the government, hospitals, and enterprises. Economic management mainly refers to the continuous improvement of economic management mode by relevant managers based on objective economic laws and aims at improving the comprehensive management level [1, 2]. This management mode needs to comprehensively strengthen the organization, implementation of economic management, and rational allocation of existing resources, so as to reduce economic costs and achieve the goal of improving economic benefits. Therefore, the comprehensive evaluation of economic management performance directly affects the survival and development of each unit and has great significance for social and economic development. Therefore, it is imperative to study an effective comprehensive evaluation method of economic management performance $[3,4]$.
At present, the research on the comprehensive evaluation method of economic management performance has made some progress. For example, Tang and Ren [5] proposed a management performance evaluation method based on the mobile network. It was decided to build a three-tiered architecture, with the first layer being the cloud platform service provider's layer, the second being the networking layer, and the third being the layer where data from the wireless sensor network would be collected and sent to the cloud platform service provider's layer. Combined with the calculation results of indicators to achieve management performance evaluation, Zhang and Liu [6] proposed a management performance evaluation method based on the ANP-matter-element model. This method focuses on improving management quality and builds a related evaluation system from the perspective of information-resource allocation. The ANP-matter-element model is built by combining the analytic hierarchy process with the matterelement model. The management performance is evaluated based on the results of index weight calculation, and the evaluation results are obtained. Chen et al. [7] proposed a 
management performance evaluation method based on the HSE perspective and Choquet integral. This approach gathers data on business economic management and performs an indepth study of the HSE management evaluation system in order to create a useful evaluation index system. Because the fuzzy correlation between evaluation indicators will create some variance in the evaluation findings, the fuzzy integral is gathered in order to develop the evaluation model and acquire the necessary management performance assessment results.

However, traditional economic management performance comprehensive evaluation methods have a low recall rate and precision, a high error rate, and a longer evaluation time. We proposed a comprehensive evaluation of economic management performance based on an improved fuzzy clustering algorithm, and the application effect of this method was verified through an experiment.

\section{Design of Comprehensive Evaluation Method for Economic Management Performance}

2.1. Data Mining Based on Improved Fuzzy Clustering Algorithm. At present, the fuzzy C-mean algorithm (FCM) is the most commonly used fuzzy clustering method, which has many advantages such as simple algorithm and easy implementation, so it has been widely used in various computer fields $[8,9]$.

Suppose there is a finite set $X=\left\{x_{1}, x_{2}, \ldots, x_{n}\right\}$, where $x_{j} \in R^{p}, j=1,2, \ldots, n$. The clustering criterion function can be expressed by the following formula:

$$
J(U, V)=\sum_{i=1}^{c} \sum_{j=1}^{n}\left(u_{i j}\right)^{m}\left\|x_{j}-v_{i}\right\|^{2},
$$

where $n$ represents the total number of samples, $c$ represents the maximum number of clustering, $m$ represents the weighted power index, $v_{i}$ represents the $i$-th clustering center, and $u_{i j}$ represents the degree to which sample $j$ belongs to the $i$-th category, satisfying $\sum_{i=1}^{c} u_{i j}=1$ [10].

FCM algorithm is to find the fuzzy classification matrix $U^{*}$ and clustering center $V^{*}$ to minimize the objective function $J(U, V)$. The iterative formula of membership degree $u_{i j}$ and clustering center $v_{i}$ in FCM algorithm can be obtained by the Lagrange multiplier method:

$$
u_{i j}=\left[\sum_{k=1}^{c}\left(\frac{\left\|x_{j}-v_{i}\right\|}{\left\|x_{j}-v_{k}\right\|}\right)^{2 / m-1}\right]^{-1}, \quad i=1,2, \ldots, c ; j=1,2, \ldots, n,
$$

$$
v_{i}=\frac{\sum_{j=1}^{n}\left(u_{i j}\right)^{m} x_{j}}{\sum_{j=1}^{n}\left(u_{i j}\right)^{m}}, \quad i=1,2, \ldots, c .
$$

The FCM algorithm makes the objective function formula (1) reach a minimum through repeated iteration of formulas (2) and (3). When formula (1) converges to the minimum value, the final classification matrix and clustering center are obtained, and the final clustering result is obtained $[11,12]$.
The traditional FCM algorithm is sensitive to the initial value of the cluster center, and the clustering effect is different with the number of clusters, and it is easy to fall into the local extremum. In order to solve this problem effectively, the improved magnetic optimization algorithm (IMOA) is used to optimize FCM [13], that is, the IMOA-FCM algorithm. IMOA-FCM algorithm is the main idea is to make data in a multidimensional space object as a fixed point and the magnetic particles as movable point, and the magnetic particles are no longer in von Neumann neighborhood structure configuration, but spread randomly in the search space, and each magnetic particle has a coding dimension of $(1, c \times$ Dim $)$ and clustering center on behalf of all of the initial value, and the code is shown in Figure 1.

The implementation process of the IMOA-FCM algorithm is as follows.

Step 1. Normalize the dataset of economic management performance for subsequent analysis.

Step 2. Determine the optimal number of clustering, as shown in Figure 2.

In Figure $2, c_{\min }$ and $c_{\max }$ represent the minimum and maximum number of clusters, respectively.

Step 3. Suppose there are $S$ membership matrices. In order to further improve the clustering accuracy, these matrices need to be initialized so as to obtain the initial value sets of the $S$ clustering centers, encode the obtained sets as the values of magnetic particles, and set the maximum iteration times to Max_itr.

Step 4. Set the initial speed value $V_{i}$ of magnetic particles to a random number between 0 and 1 .

Step 5. Obtain the magnetic field intensity of each magnetic particle through the objective function of the FCM algorithm $B_{i}$ as follows:

$$
\begin{aligned}
b_{i}(t)= & \frac{\operatorname{fit}_{i}(t)-\operatorname{worst}(t)}{\operatorname{best}(t)-\operatorname{worst}(t)}, \\
& \left\{\begin{array}{l}
\operatorname{best}(t)=\min _{i \in\{1,2, \ldots, S\}} \mathrm{fit}_{i}(t), \\
\operatorname{worst}(t)=\max _{i \in\{1,2, \ldots, S\}} \mathrm{fit}_{i}(t),
\end{array}\right. \\
B_{i}= & \frac{b_{i}(t)}{\sum_{j=1}^{S} b_{j}(t)},
\end{aligned}
$$

where $\mathrm{fit}_{i}(t)$ is the objective function value of the $i$-th magnetic particle in the $t$-th iteration.

Step 6. Calculate the mass value of each magnetic particle:

$$
M^{\text {itr }}=\alpha+\rho \times B^{\text {itr }},
$$

where $\alpha$ and $\rho$ are constants.

Step 7. Calculate the distance between the two magnetic particles. 


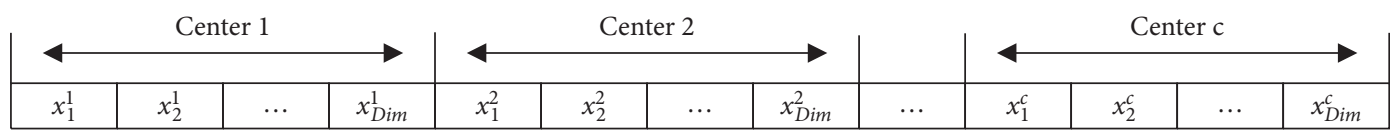

FIgURE 1: Coding of a single magnetic particle.

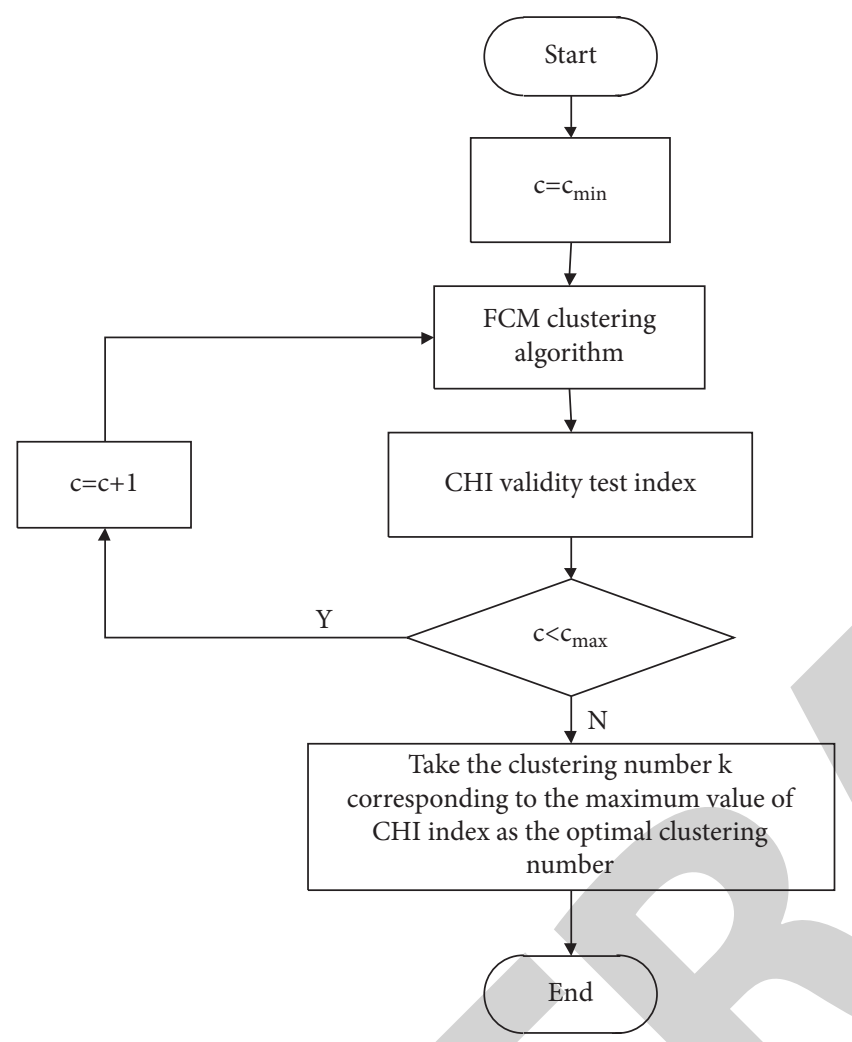

Figure 2: Process of obtaining the optimal number of clusters for $\mathrm{CHI}$ index.

Suppose $D$ is the distance between the magnetic particle $i$ and adjacent magnetic particle $j$, and the formula is as follows:

$$
D\left(X_{j}^{\mathrm{itr}}, X_{i}^{\mathrm{itr}}\right)=\frac{1}{\operatorname{Dim}} \sum_{r=1}^{\operatorname{Dim}} \mid \frac{X_{j{ }^{\prime} r}^{\mathrm{itr}}-X_{i}{ }^{\mathrm{itr}}}{\text { value }_{-} l_{r}-\text { value }_{-} h_{r}}
$$

Step 8. Calculate the resultant force on each magnetic particle as follows:

$$
\text { force }_{i}=G \cdot \sum_{j=1, j \neq S}^{S} \operatorname{rand} \cdot \frac{\left(X_{j}-X_{i}\right) \cdot B_{j}}{D},
$$

where $G$ is the resultant force adjustment factor, whose calculation formula is as follows, and $G_{0}$ is the update factor of $G$ :

$$
G=G_{0} \cdot e^{-\sigma \cdot \operatorname{itr} / \text { Max_itr }},
$$

where $G_{0}$ is the initial value of search adjustment and $\sigma$ is the attenuation coefficient.

Step 9. Update the acceleration, velocity, and position values of magnetic particles as follows:

$$
\begin{aligned}
V_{i}(t+1)= & \operatorname{rand} \cdot V_{i}(t)+c_{1} \operatorname{rand}_{2}\left[\text { pbest }-X_{i}(t)\right] \\
& +c_{2} \operatorname{rand}_{3}\left[\text { gbest }-X_{i}(t)\right]+\frac{\text { force }_{i}}{M_{i}}, \\
X_{i}(t+1)= & X_{i}(t)+V_{i} .
\end{aligned}
$$

Step 10. Recalculate the target value function according to the position of the new magnetic particle, and judge whether the number of iterations at this time meets Max_iter. If so, output the data mining results of economic management performance; otherwise, repeat Steps 5-9.

2.2. Comprehensive Evaluation of Economic Management Performance. According to scientific, feasible, and other principles, and after studying the research results of many experts, this paper mainly constructs the comprehensive evaluation index system of economic management performance, as shown in Table 1.

In this paper, analytic hierarchy process is used to calculate the weight of evaluation indicators, and the specific process is as follows.

2.2.1. Architecture Hierarchy Model. The elements in the decision-making problem are stratified, and the structure and association of each level are displayed in the form of charts. If the elements in a certain level are still very complicated, they can be further divided into levels. In this paper, the index system is divided into two layers $[14,15]$.

2.2.2. Architectural Judgment Matrix. The importance measurement method of evaluation indicators usually uses the method of 1-9 and its reciprocal for statistics, and the scale table is shown in Table $2[16,17]$.

The judgment matrix is expressed by the following formula:

$$
P=\left(P_{i j}\right)(i=1,2, \ldots, n ; j=1,2, \ldots, n) .
$$

2.2.3. Using the Sum-Product Method to Complete the Single Hierarchical Sorting. Normalized transformation of each column of $P$ is given by

$$
\bar{P}_{i j}=\frac{p_{i j}}{\sum_{k=1}^{n} p_{k j}} \quad(i=1,2, K, n ; j=1,2, K,, n) .
$$

Find the sum of elements of $P$ line $\bar{W}_{i}$ : 
TABLE 1: Evaluation index system.

\begin{tabular}{lc}
\hline Level indicators & Secondary indicators \\
\hline Profitability & $\begin{array}{c}\text { Return on investment } \\
\text { Return on equity }\end{array}$ \\
& $\begin{array}{c}\text { Value preservation and appreciation rate of capital } \\
\text { Receivable turnover rate } \\
\text { Asset turnover } \\
\text { Inventory turnover } \\
\text { Cperation ability }\end{array}$ \\
Current ratio \\
Debt paying ability & Debt-to-equity ratio \\
Development capacity & Interest coverage multiple \\
& Asset-to-liability ratio \\
Sales growth rate \\
\hline
\end{tabular}

TABLE 2: Scale values.

\begin{tabular}{lc}
\hline$P_{i j}$ & Comparison of $P_{i}$ and $P_{j}$ in importance \\
\hline 1 & $P_{i}$ and $P_{j}$ are equally important \\
3 & $P_{i}$ is slightly more important than $P_{j}$ \\
5 & $P_{i}$ is obviously more important than $P_{j}$ \\
7 & $P_{i}$ is more strongly important than $P_{j}$ \\
9 & $P_{i}$ is more important than $P_{j}$ \\
$2,4,6,8$ & Between the above two adjacent judgments \\
\hline
\end{tabular}

$$
\bar{W}_{i}=\sum_{j=1}^{n} P_{i j}
$$

$W_{i}$ is obtained after normalization of $\bar{W}_{i}$ :

$$
W_{i}=\frac{\bar{W}_{i}}{\sum_{i=1}^{n} \bar{W}_{i}}
$$

The obtained $W_{1}, W_{2}, \ldots, W_{n}$ is the weight of the elements after the single ranking.

2.2.4. Consistency Verification. Due to the subjective influence of the evaluator in the measurement process, it is difficult to reach an agreement. In such cases, consistency test can be carried out according to the principle of the specific formula of the matrix to ensure that the judgment results are relatively consistent on the whole [18]:

$$
\mathrm{CI}=\frac{\lambda_{\max }-n}{n-1},
$$

where $n$ represents the order of the matrix. When $C I=0$, the judgment matrix $P$ is completely consistent, but such a situation is difficult to happen. In practice, the closer $\lambda_{\max }$ is to $n$, the smaller the value of $C I$ is, and the better the consistency of $P$ matrix is $[19,20]$.

The evaluation criterion is as follows: when $C_{R}=\mathrm{CI} / R_{\mathrm{i}}<0.1$, it means that the $P$ matrix has satisfactory consistency; otherwise, the $P$ matrix should be adjusted until satisfactory consistency appears. The maximum value of the judgment matrix is calculated, then formula (14) is used to calculate $\mathrm{CI}$, and finally, $C_{R}$ is calculated for consistency judgment.
After completing the hierarchical single ranking of the weight values of the elements at the upper level, all the elements also need to conduct an incremental total ranking of the relative weight of the final evaluation objective. Its relative weight is calculated from top to bottom and merged layer by layer.

Then, the total ranking of the elements at layer $k$ relative to the total evaluation objective is

$$
W_{i}^{k}=\sum_{j=1}^{m} P_{i j}^{k} W_{j}^{k} \quad(i=1,2, K, n) .
$$

Based on the calculation results of index weight, the BP neural network was optimized by genetic algorithm, and the comprehensive evaluation model of economic management performance was established to obtain the relevant evaluation results. Because the number of input layer and output layer nodes is determined by the specific question, in this paper, the knowledge management performance evaluation is determined by determining the 12 evaluation indexes, so the neural network model with 12 input indicators is adopted; that is, there are 12 nodes in the input layer and only one neuron in the output of the network layer, to generate the numerical representation of the comprehensive evaluation results for knowledge management. Its value ranges from 0 to 1 . The realization process of the BP neural network algorithm is as follows:

(1) Assign a small random number between $[-1,1]$ to the weights and thresholds of the network

(2) Input a learning sample $X_{k}$ to calculate the output value of each node of the hidden layer:

$$
y_{j}=f\left(\sum_{i=0}^{n} v_{i j} x_{i}\right) \quad(j=1,2, \ldots, m) .
$$

(i) Calculate the output value of nodes in the output layer [21, 22] as follows:

$$
o_{k}=f\left(\sum_{j=0}^{m} w_{j k} y_{j}\right) \quad(k=1,2, \ldots, l) .
$$

(3) Calculate the correction of connection weights between nodes of the output layer and nodes of the hidden layer as follows: 


$$
\Delta w_{j k}=\eta \delta_{k}^{o} y_{j}=\eta\left(d_{k}-o_{k}\right) o_{k}\left(1-o_{k}\right) .
$$

(4) Calculate the correction of connection weights between nodes of the hidden layer and nodes of the input layer as follows:

$\Delta v_{i j}=\eta \delta_{j}^{y} x_{i}=\eta\left(\sum_{k=1}^{l} \delta_{k}^{o} w_{j k}\right) y_{j}\left(1-y_{j}\right) x_{i}$.

(5) Use error correction to correct weights and thresholds of the network $[23,24]$ :

$$
\begin{gathered}
w_{j k}(t+1)=w_{j k}(t)+\Delta w_{j k}, \\
v_{i j}(t+1)=v_{i j}(t)+\Delta v_{i j} .
\end{gathered}
$$

(6) Analyze whether the error function $E$ is less than the expected error accuracy. If so, end the algorithm; otherwise, return to step (2). $o_{k}$ is the desired output [25].

Because the BP neural network's generalization ability is poor, its output error rises. To handle this issue efficiently, this research employs a genetic algorithm to optimize the network, as well as a training sequence of selection, crossover, mutation, and BP network to maintain the best individual and compute the performance function. The whole procedure is terminated if the mistake fits the criteria; else, the genetic operation process is repeated until the end condition is reached. The algorithm's flow is shown in Figure 3.

\section{Experimental Design}

In order to verify the effectiveness of the comprehensive evaluation of economic management performance based on the improved fuzzy clustering algorithm designed in this paper, an experimental design is carried out. The overall experimental process is as follows.

In order to ensure that the simulation experiment results can be as close to the actual situation as possible, it is necessary to ensure that the experiment is carried out in the same environment. The overall experimental environment settings are shown in Table 3.

Using reference [5] method and reference [6] method, which were adopted to evaluate the recall rate and precision of data mining for economic management performance and evaluate the error rate and time-consuming of management performance comprehensively, we compared the four indicators to verify the application effect of different methods.

3.1. Recall Rate. The recall rate of economic management performance data mining of reference [5] method, reference [6] method, and this method is compared, and the comparison results are shown in Figure 4.

The analysis of the data in Figure 4 shows that recall rates of different methods show a trend of fluctuation with the increase of the number of experiments. The maximum recall rate of reference [5] method is $84 \%$, and the minimum recall rate is $72 \%$. The maximum recall rate of reference [6] method is $83 \%$, and the minimum is $66 \%$. Compared with the two methods, the maximum recall rate of the proposed method is $97 \%$ and the minimum value is $95 \%$, indicating that the proposed method has a higher recall rate of economic management performance data mining, indicating that the mining results of the proposed method are more comprehensive.

3.2. Accuracy. Comparison is made between reference [5] method, reference [6] method, and this paper's method in the accuracy rate of economic management performance data mining. The comparison results are shown in Figure 5.

By analyzing the data in Figure 5, it can be seen that, with the increase of the number of experiments, the accuracy of different methods showed a trend of fluctuation. Reference [5] approach had a maximum accuracy of 85 percent and a minimum accuracy of 59 percent among them. Reference [6] approach had a maximum accuracy of 88 percent and a minimum accuracy of 63 percent. When compared to the two methods, the method in this paper has a maximum accuracy of 98 percent and a minimum accuracy of 95 percent, indicating that the method in this paper has a higher accuracy of economic management performance data mining, indicating that the method's mining results are more accurate.

3.3. Assessment Error Rate. Reference [5] method, reference [6] method, and this paper's method are compared for the error rate of the comprehensive evaluation of economic management performance. The comparison results are shown in Figure 6.

The analysis of the data in Figure 6 shows that the evaluation error rate of different methods changes with the increase of the number of experiments. Among them, the comprehensive evaluation error rate of economic management performance of reference [5] method varies between $-15 \%$ and $17 \%$, and that of reference [6] method varies between $-22 \%$ and $14 \%$. Compared with the two methods, the error rate of the comprehensive evaluation of economic management performance in this method varies between $-3 \%$ and $4 \%$, and the error rate of the comprehensive evaluation of economic management performance in this method is lower, indicating that this method can achieve accurate evaluation of economic management performance.

3.4. Evaluation Time. The time consumption of a comprehensive evaluation of economic management performance of reference [5] method, reference [6] method, and this method is compared, and the comparison results are shown in Table 4.

By analyzing the data in Table 4, it can be seen that the average time consumption of the comprehensive evaluation of economic management performance of reference [5] method is $3.42 \mathrm{~s}$, the average time consumption of the comprehensive evaluation of economic management performance of reference [6] method is $1.51 \mathrm{~s}$, and the average 


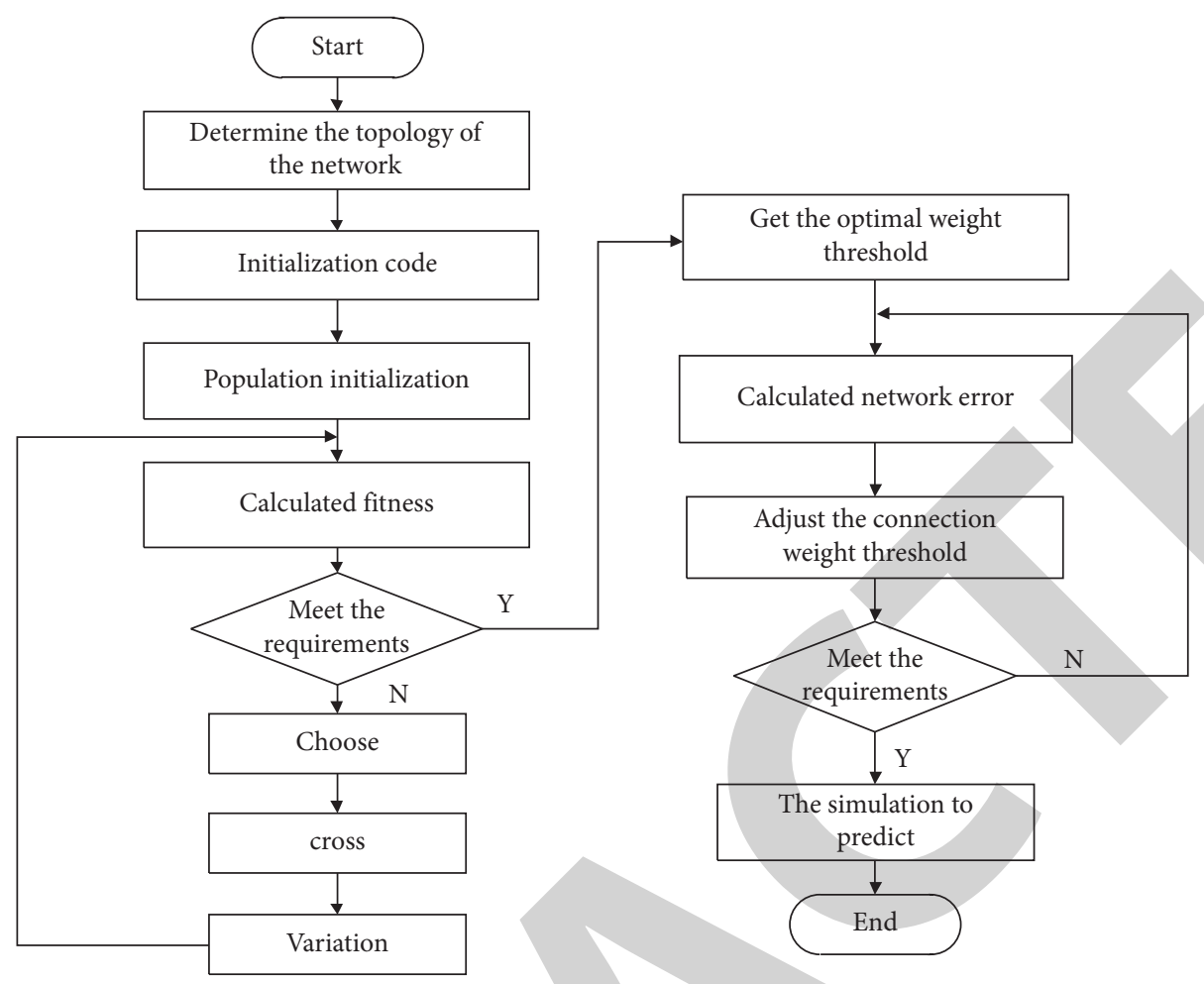

Figure 3: Optimization process of the BP neural network.

TABLE 3: Experimental environment settings.

\begin{tabular}{lc}
\hline Name & Parameter \\
\hline Central processing unit & Intel (R) Core (TM) 2 Duo CPU E7500@2.93 GHz 2.94 GHz \\
Install memory & $8.00 \mathrm{G}$ \\
Display adapter & NVIDIA GeForce GTX 550 Ti \\
Development tool & Visual Studio 2010 \\
Language and corresponding library & C/C++, OpenCV 2.4.3, Qt4.7.4, Matlab R20I3a \\
System environment & Windows 10 \\
Simulation software & Matlab 7.2 \\
\hline
\end{tabular}

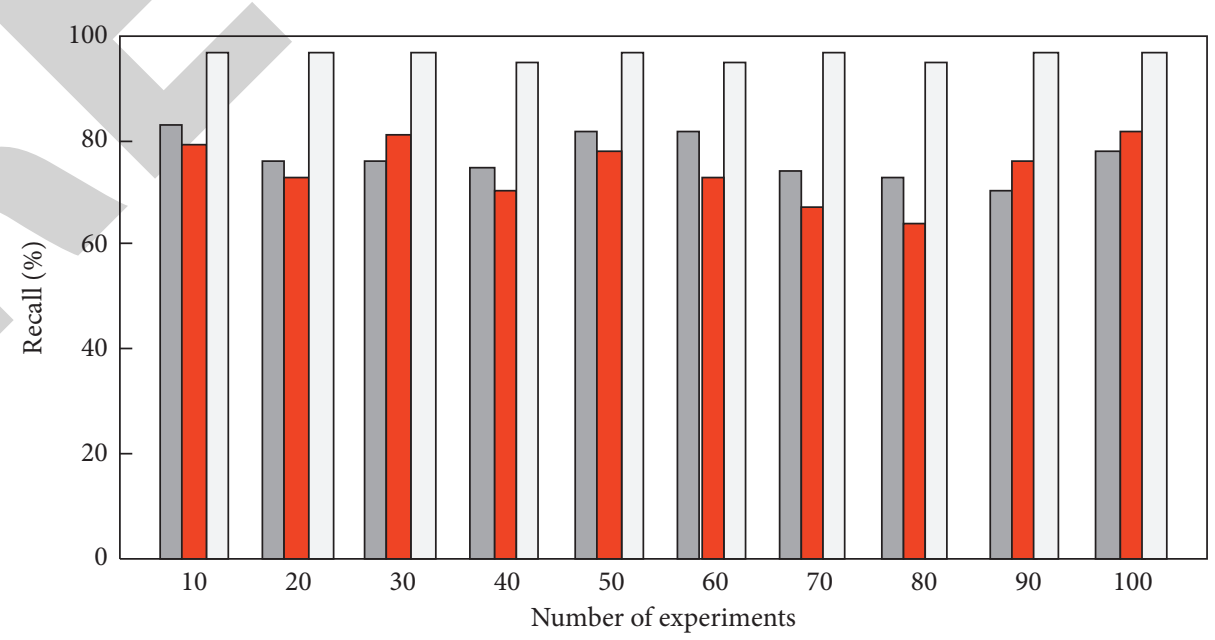

Reference [5] method

Reference [6] method

Method of this paper

FiguRE 4: Comparison of recall rates. 


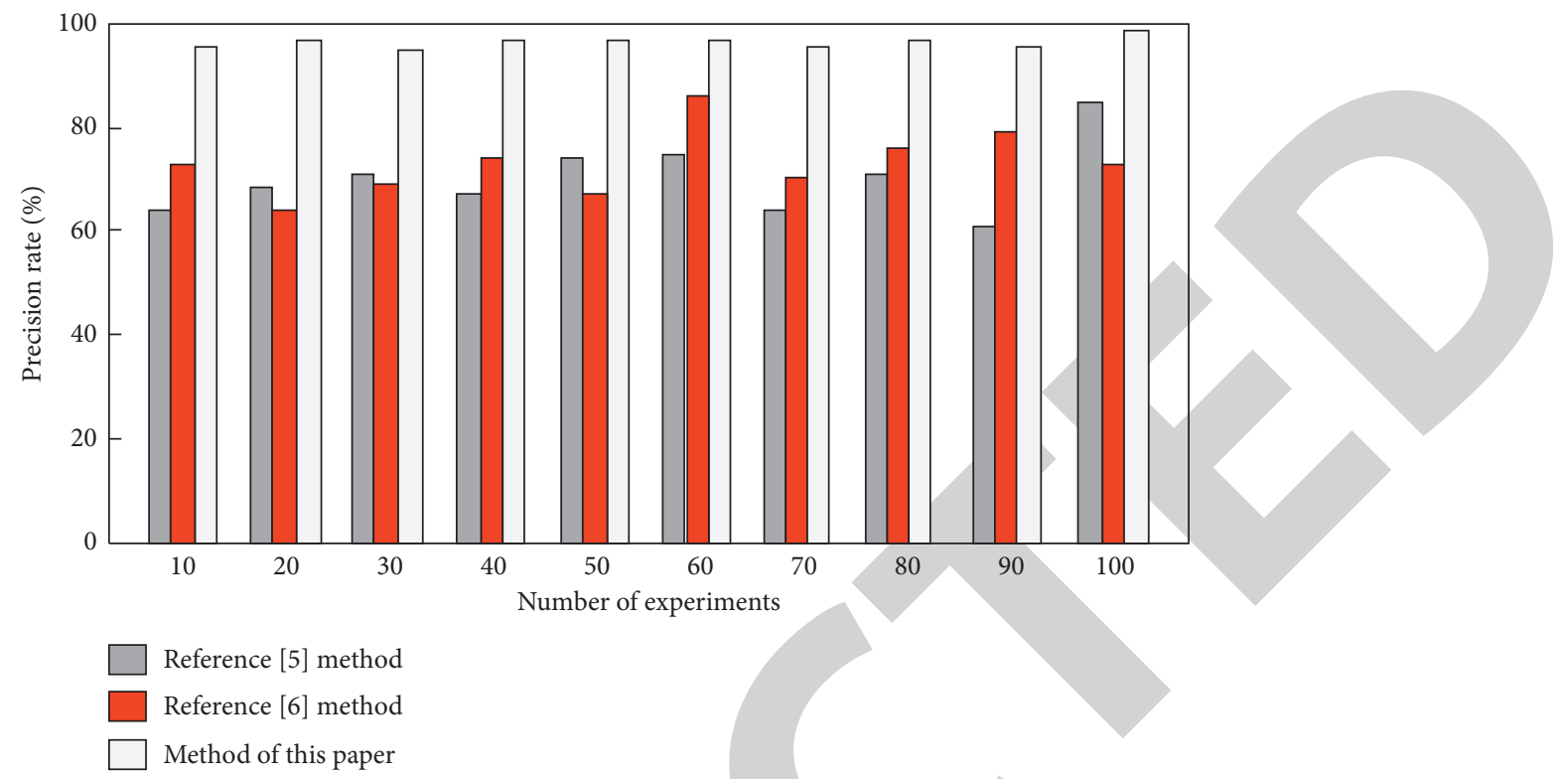

FIgURE 5: Comparison of accuracy.

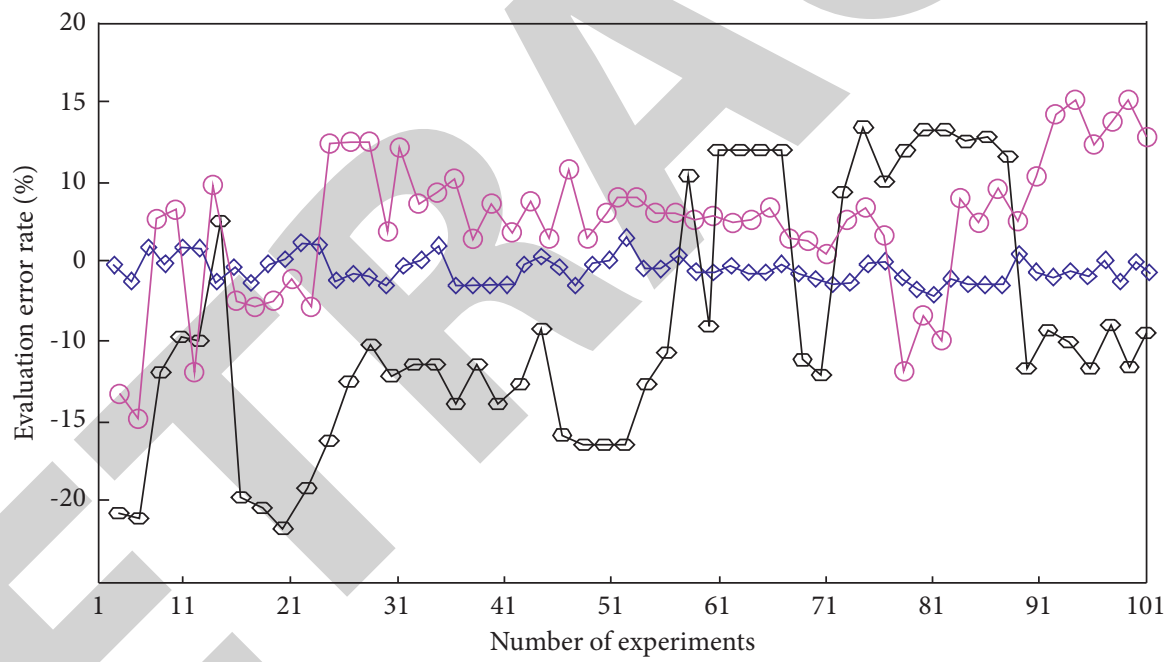

- Reference [5] method

$\square$ Reference [6] method

$\checkmark$ Method of this paper

Figure 6: Assessment error rate.

TABLE 4: Comparison of evaluation time (unit: s).

\begin{tabular}{lccc}
\hline Number of experiments & Reference [5] method & Reference [5] method & Method in this paper \\
\hline 10 & 3.65 & 1.63 & 0.96 \\
20 & 3.47 & 1.47 & 0.85 \\
30 & 2.58 & 1.58 & 0.74 \\
40 & 3.64 & 1.24 & 0.85 \\
50 & 4.84 & 1.36 & 0.93 \\
60 & 2.55 & 1.47 & 0.74 \\
70 & 3.47 & 1.48 & 0.81 \\
80 & 3.41 & 1.59 & 0.76 \\
90 & 3.64 & 1.33 & 0.68 \\
100 & 2.96 & 1.96 & 0.74 \\
Average value & 3.42 & 1.51 & 0.81 \\
\hline
\end{tabular}


time consumption of the comprehensive evaluation of economic management performance of this method is $0.81 \mathrm{~s}$. The evaluation time of this method is longer than that of reference [5] and reference [6] method shortens $2.61 \mathrm{~s}$ and $0.7 \mathrm{~s}$, which shows that the comprehensive evaluation of economic management performance of this method takes less time and is more efficient.

\section{Conclusion}

With the rapid development of the social economy, the current economic management concept has been widely applied in all walks of life, promoting industry to further improve economic benefit, and for economic management performance evaluation, the level of economic management and performance can be clearly understood, and further adjustments for economic management were made based on the related research results, so the performance comprehensive evaluation method of economic management has been developed. Simulation results show that, in the proposed method, the maximum recall rate was $97 \%$ and the minimum was $95 \%$. The maximum accuracy is $98 \%$, and the minimum is $95 \%$, indicating that the proposed method's economic management performance mining effect is better. Moreover, the error rate of the comprehensive evaluation of economic management performance of the method in this paper varies between $-3 \%$ and $4 \%$, and the average evaluation time is $0.81 \mathrm{~s}$, indicating that the method in this paper can be used to quickly obtain accurate performance evaluation results of economic management, which can be more widely used in practice.

\section{Data Availability}

The data used to support the findings of this study are included within the article.

\section{Conflicts of Interest}

The authors declare that they have no conflicts of interest.

\section{References}

[1] K. Kim, "The interplay between the social and economic human resource management systems on innovation capability and performance," International Journal of Innovation Management, vol. 25, no. 07, pp. 2150074-2150086, 2021.

[2] Z. Ling, B. Wz, A. Zc, Y. Hu, U. Schmidhalter, and X. Chen, "Environmental, human health, and ecosystem economic performance of long-term optimizing nitrogen management for wheat production," Journal of Cleaner Production, vol. 311, no. 15, pp. 127620-127631, 2021.

[3] J. Mariyono, "Improvement of economic and sustainability performance of agribusiness management using ecological technologies in Indonesia," International Journal of Productivity and Performance Management, vol. 69, no. 5, pp. 9891008, 2019.

[4] A. Dehghani, F. Khaleghi, and S. Sanzighi, "Investigating and ranking the components of supply chain management performance in economic projects based on the hybrid fuzzy
ANP-based approach, fuzzy topsis," Journal of Economics and Economic Education Research, vol. 20, no. 1, pp. 15-26, 2019.

[5] S. Tang and C. H. Ren, "Laboratory management performance evaluation system based on mobile network," Modern Electronic Technology, vol. 44, no. 13, pp. 75-79, 2021.

[6] Y. L. Zhang and M. Y. Liu, "Research on emergency management performance evaluation model based on ANP matter-element model," Journal of Wuhan University of Technology, vol. 42, no. 1, pp. 6-11, 2020.

[7] W. G. Chen, H. H. Wang, and S. X. Zhang, "Research on performance evaluation of large-scale engineering project management based on HSE perspective and Choquet score," Architectural Technology, vol. 50, no. 6, pp. 707-710, 2019.

[8] S. b Yue, Q. h Wang, and X. c Wang, "Cloud information storage encryption simulation based on fusion fuzzy clustering algorithm," Computer Simulation, vol. 37, no. 3, pp. 449-452, 2020.

[9] D. Mújica-Vargas, "Superpixels extraction by an Intuitionistic fuzzy clustering algorithm," Journal of Applied Research and Technology, vol. 19, no. 2, pp. 141-153, 2021.

[10] Y. Gong, S. Lin, F. He, Y. He, and J. Song, "Damage identification of prefabricated reinforced concrete box culvert based on improved fuzzy clustering algorithm and acoustic emission parameters," Advances in Materials Science and Engineering, vol. 2021, no. 7, 13 pages, Article ID 6660915, 2021.

[11] K. Jalaldeen, M. Malathi, P. Sinthia, M. Vadivel, and B. Maruthi Shankar, "An automatic identification of lung tumor by using CNN network and fuzzy-clustering algorithm," Materials Today Proceedings, vol. 45, no. 1, pp. 2921-2924, 2021.

[12] F. Zhao, Z. Zeng, H. Liu, R. Lan, and J. Fan, "Semisupervised approach to surrogate-assisted m kernel intuitionistic fuzzy clustering algorithm for color image segmentation," IEEE Transactions on Fuzzy Systems, vol. 28, no. 6, pp. 1023-1034, 2020.

[13] L. Houcine, M. Bouzbida, and A. Chaari, "Improved type2NPCM fuzzy clustering algorithm based on adaptive particle swarm optimization for takagi-sugeno fuzzy modeling identification," International Journal of Fuzzy Systems, vol. 22, no. 4, pp. 1-10, 2020.

[14] R. Bornéo, M. M. Franco, B. C. Orlandin, N. Moraes, and L. Corso, "Application of analytic hierarchy process considering artificial neural network and ARIMA for selecting a chemical waste plant," Scientia cum Industria, vol. 9, no. 1, pp. 30-37, 2021.

[15] H. Ding, J. Wang, W. Liao, T. Liang, and H. Dai, "Site selection of self-driving and recreational vehicle camps in China:An investigation using analytic hierarchy process and entropy," Journal of Transportation Engineering: English version, vol. 8, no. 5, pp. 16-25, 2021.

[16] X. Liu, H. Liu, Z. Wan, H. Pei, and H. Fan, "The comprehensive evaluation of coordinated coal-water development based on analytic hierarchy process fuzzy," Earth Science India, vol. 14, no. 1, pp. 311-320, 2021.

[17] D. Selvam, S. Maitra, P. Parthiban, and A. Z. Hameed, "Composite techniques of structural equation modeling and analytic hierarchy process for information technology vendor selection," International Journal of Information Technology and Decision Making, vol. 20, no. 4, pp. 1-35, 2021.

[18] A. Srivastava, S. K. Gaur, S. Swami, and D. K. Banwet, "Analysis of interpretive structural model of Indian railway security system by analytic hierarchy process (AHP)," Journal of Advances in Management Research, vol. 16, no. 3, pp. 378-397, 2019. 
[19] L. A. Ocampo, "Decision modeling for manufacturing sustainability with fuzzy analytic hierarchy process," Global Business Review, vol. 20, no. 1, pp. 25-41, 2019.

[20] N. Elshaboury, "Prioritizing risk events of a large hydroelectric project using fuzzy analytic hierarchy process," Journal of Project Management, vol. 6, no. 3, pp. 107-120, 2021.

[21] X. Zhou, F. U. Xuyun, M. Zhao, and S. Zhong, "Regression model for civil aero-engine gas path parameter deviation based on deep domainadaptation with Res-BP neural network," Chinese Journal of Aeronautics, vol. 34, no. 1, pp. 12-21, 2021.

[22] Y. Liu, X. Wang, X. Zhu, and Y. Zhai, "Thermal error prediction of motorized spindle for five-axis machining center based on analytical modeling and BP neural network," Journal of Mechanical Science and Technology, vol. 35, no. 1, pp. 281-292, 2021.

[23] F. Liu, B. Han, W. Qin, L. Wu, and S. Li, "A prediction method of urban water pollution based on improved BP neural network," International Journal of Environmental Technology and Management, vol. 24, no. 1, pp. 17-25, 2021.

[24] H. Wang, M. Hong, and Z. Hong, "Research on BP neural network recommendation model fusing user reviews and ratings," IEEE Access, vol. 9, pp. 86728-86738, 2021.

[25] Z. Shen, "Performance analysis of bf cement-based composites for building based on BP neural network," Key Engineering Materials, vol. 852, no. 1, pp. 209-219, 2020.

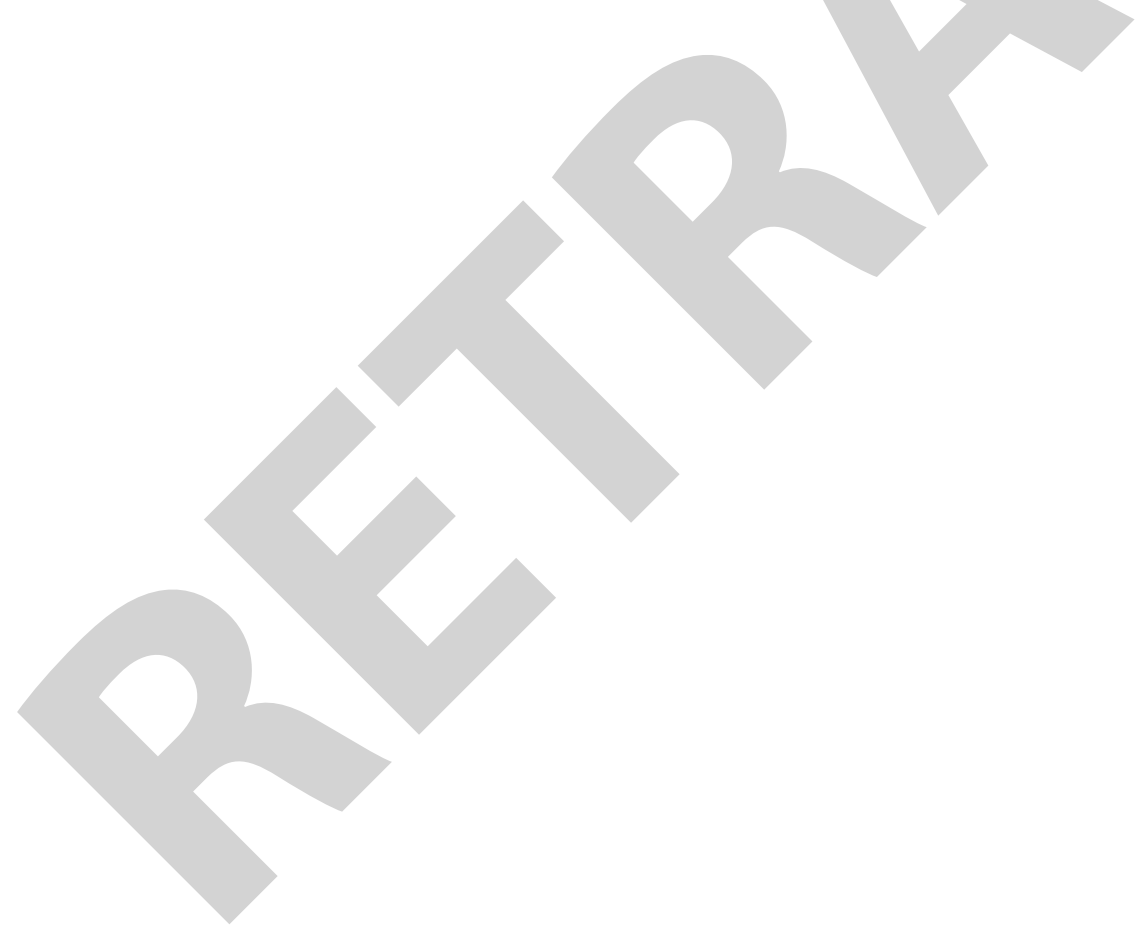

